\title{
Recombination dynamics in ZnO nanowires: Surfaces states versus mode quality factor
}

\author{
J. S. Reparaz, ${ }^{1, a)}$ F. Güell, ${ }^{2}$ M. R. Wagner, ${ }^{1}$ G. Callsen, ${ }^{1}$ R. Kirste,,${ }^{1}$ S. Claramunt, ${ }^{2}$ \\ J. R. Morante, ${ }^{2,3}$ and A. Hoffmann ${ }^{1}$ \\ ${ }_{1}^{1}$ Institut für Festkörperphysik, Technische Universität Berlin, Hardenbergstr. 36, 10623 Berlin, Germany \\ ${ }^{2}$ Departament d'Electrònica, M-2E, IN2UB, Universitat de Barcelona, C/Martí i Franquès 1, 08028 \\ Barcelona, Catalunya, Spain \\ ${ }^{3}$ Institut de Recerca en Energia de Catalunya (IREC), C/Josep Pla 2, 08019 Barcelona, Catalunya, Spain
}

(Received 11 July 2010; accepted 11 September 2010; published online 1 October 2010)

\begin{abstract}
In this work, we investigate the influence of finite size on the recombinations dynamics of $\mathrm{ZnO}$ nanowires. We demonstrate that diameter as well as length of nanowires determine the lifetime of the neutral donor bound excitons. Our findings suggest that while the length is mainly responsible for different mode quality factors of the cavity-like nanowires, the diameter determines the influence of surface states as alternative recombinations channels for the optical modes trapped in the nanocavity. In addition, comparing nanowires grown using different catalyst we show that the surfaces states strongly depend on each precursor characteristics. (C) 2010 American Institute of Physics. [doi:10.1063/1.3496444]
\end{abstract}

The great amount of $\mathrm{ZnO}$ nanostructures recently produced such as nanodots, nanocrystals, tetrapods, nanorods, and nanowires (NWs) have opened an entirely new field of research activities. ${ }^{1}$ In particular, $\mathrm{ZnO}$ NWs represent an extremely convenient system since it is the lowest dimensional system where electrical conductivity measurements can be performed. A giant exciton-polariton coupling has been theoretically suggested for $\mathrm{ZnO}$ quantum dots, ${ }^{2}$ and recently experimentally demonstrated for a single $\mathrm{ZnO} \mathrm{NW}{ }^{3}$ The longitudinal-to-transverse exciton-polariton splitting $\left(\Delta \omega_{L T}\right)$ was shown to be as high as $164 \mathrm{meV}$ for $\mathrm{ZnO} \mathrm{NWs}$, i.e., almost two orders of magnitude larger than in bulk samples $\left(\Delta \omega_{L T} \approx 2 \mathrm{meV}\right.$ for the free A exciton-polariton). This result reflects the great influence of finite-size on the excitonpolariton coupling and, consequently, represents a strong motivation for a detailed study of finite-size effects on the cavity-like properties of these nanostructures.

The large surface-to-volume ratio of this one dimensional system is responsible for the high interaction between core and surface electronic states. In addition, the shape of the NWs constitutes itself an optical nanocavity for the propagating electromagnetic waves generated by optical or even electrical excitation. In a recent work, ${ }^{4}$ we have shown that the recombination dynamics of the donor bound excitons (DX) are influenced by the NW's diameter. This effect was attributed to the presence of size-dependent surface states. Nevertheless, due to the nature of the investigated samples we were not able to provide any hint of the influence of finite size on mode quality factor $(Q)$ of the nanocavities which are formed by the NWs. For this purpose, the DX are an ideal candidate since these are spatially localized states. Consequently, the optical properties of the DX as well as their recombination dynamics provide direct information about the influence of the NW's size on the confinement of the optical modes.

The main purpose of this paper is to show that the cavity-like properties of $\mathrm{ZnO} \mathrm{NWs}$ are determined by two

${ }^{a)}$ Electronic mail: jsreparaz@gmail.com. main contributions as follows: (i) the influence of surfaces states, and (ii) the $Q$-factor of the cavity composed by the NWs. We discuss the relative influence of these effects in NWs with different dimensions and we show that while the $Q$-factor is mainly governed by the NW's length, additional recombination channels are introduced as surface states by decreasing the NW's diameter. Comparing NWs with similar dimensions grown from different precursors, we show that the surface states strongly depend on the characteristics of each particular precursor.

In order to separately investigate the influence of surface states to that of the $Q$-factor we have grown two different set of samples (three samples for each set) using different precursors for the NW's nucleation. The two different catalysts for each set of samples consisted of a thin film of sputtered $\mathrm{Au}(\mathrm{s}-\mathrm{Au})$ and colloidal $\mathrm{Au}(\mathrm{c}-\mathrm{Au})$ nanoparticles, respectively. All the samples were grown using the vapor-liquidsolid method (see Ref. 5 for a detailed description). The s-Au NWs were purposely designed to investigate the influence of the diameter on the recombination dynamics and consisted of three samples with diameters of $\mathrm{d}=50,85,130 \mathrm{~nm}(L$ $\approx 1 \mu \mathrm{m})$. The c-Au NWs were employed to study the length $(L)$ dependence and resulted in $L=1.7,2.4$, and $3.5 \mu \mathrm{m}(\mathrm{d}$ $\approx 90 \mathrm{~nm}$ ). The optical properties of these nanostructures were investigated using a micro-photoluminescence setup. The excitation energy was set to $3.496 \mathrm{eV}(355 \mathrm{~nm})$ and focused onto the samples using a $63 \times(\mathrm{NA}=0.75)$ microscope objective mounted on a XYZ piezoelectric stage, which allowed steps of $100 \mathrm{~nm}$. The spatial resolution of the system is estimated to be about $300 \mathrm{~nm}$. For the timeresolved measurements we have used the time correlated single-photon counting technique with a time resolution of about 20 ps.

In Figs. 1(a)-1(c) we show field-emission scanning electron microscope (FESEM) images of s-Au $\mathrm{ZnO} N W$ s with mean diameters of $\mathrm{d}=50 \mathrm{~nm}, 85 \mathrm{~nm}$, and $130 \mathrm{~nm}$, respectively. The diameter of the NWs was tailored changing the thickness of the s-Au thin layer previous to the $\mathrm{ZnO}$ deposition. The fact that the NWs grow almost horizontally aligned 


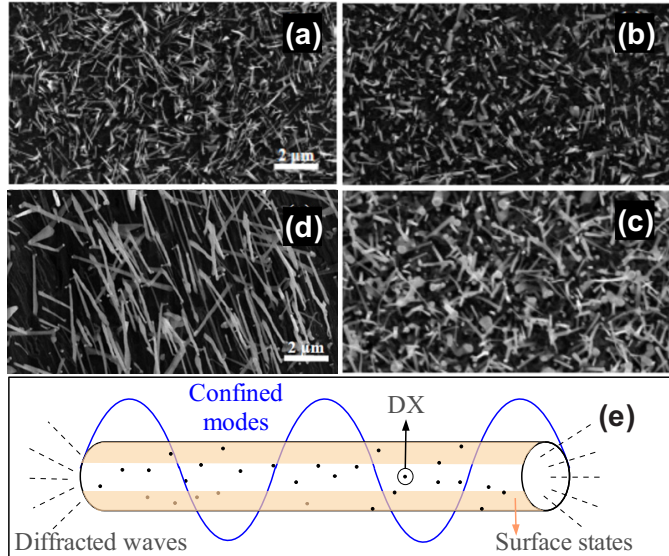

FIG. 1. (Color online) FESEM images of four samples with ZnO NWs of different diameters of (a) $50 \mathrm{~nm}$, (b) $85 \mathrm{~nm}$, and (c) $130 \mathrm{~nm}$ grown using sputtered $\mathrm{Au}$ as catalyst, and (d) a representative image of a sample grown using Au colloids. A scheme of a single NW showing its cavity-like properties and the presence of surface states is shown in (e).

is due to the lattice mismatch with the $\mathrm{SiO}_{2} / \mathrm{Si}$ substrate. For the present experiments this situation represents an advantage since in combination with the high spatial resolution of the micro-photoluminescence setup $(\approx 300 \mathrm{~nm})$ it allows to probe only a few NWs within the laser spot. In Fig. 1(d) we show a representative image of a c-Au $\mathrm{ZnO}$ NWs sample. A scheme of a single NW showing the presence of surface states, DX states, and the optical modes confined in a NW is shown in Fig. 1(e).

We show in Fig. 2 the photoluminescence spectra of the s-Au and c-Au NWs at $4 \mathrm{~K}$. All the samples exhibit a broad transition around $3.377 \mathrm{eV}$ which arises from the freeexciton recombination $\left(\mathrm{FX}_{A}\right)$. Nevertheless, the dominant features of these spectra mainly originate from the neutral-DX around $3.360 \mathrm{eV}$. Finally, in the lower energy region a broad transition around $3.314 \mathrm{eV}$ is observed only for the s-Au NWs, which probably arises from a free-tobound recombination (e, A) as recently suggested by Schirra et al. ${ }^{6}$ Concerning the DX lines, the two lines around 3.357 $\mathrm{eV}$ and $3.361 \mathrm{eV}$ are identified as arising from $\mathrm{In}\left(\mathrm{I}_{9}\right)$ and $\mathrm{Al}$ ( $\left.\mathrm{I}_{6}\right)$ impurities, respectively. ${ }^{7}$ The origin of the emission at

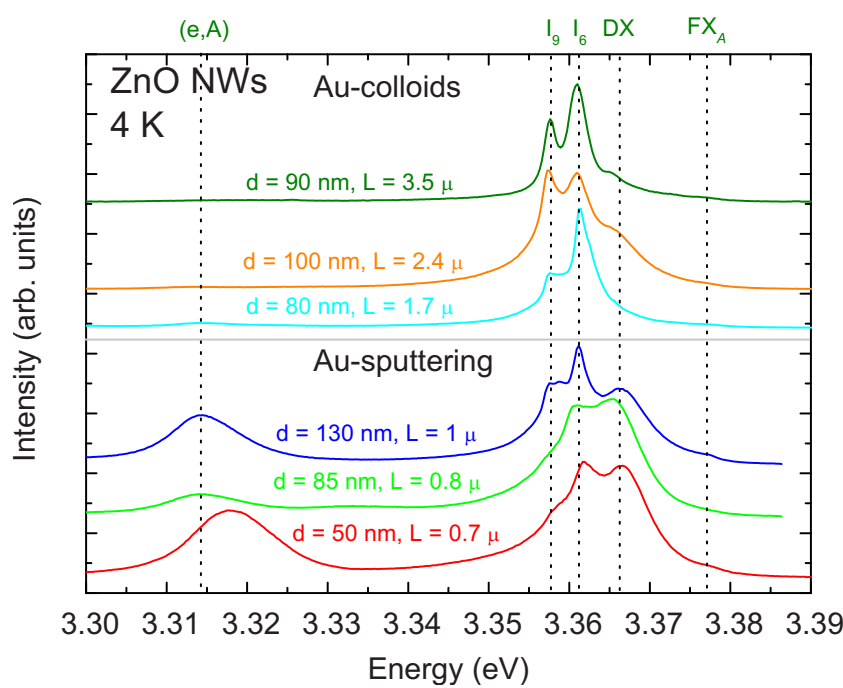

FIG. 2. (Color online) Low temperature photoluminescence spectra for the $\mathrm{s}-\mathrm{Au}$ and c-Au $\mathrm{ZnO}$ NWs with different diameters and lengths. The vertical dotted lines show the position of the $\mathrm{FX}_{\mathrm{A}}(3.377 \mathrm{eV}), \mathrm{DX}(3.366,3.361$, $3.357 \mathrm{eV})$, and $(\mathrm{e}, \mathrm{A})(3.314 \mathrm{eV})$ transitions.

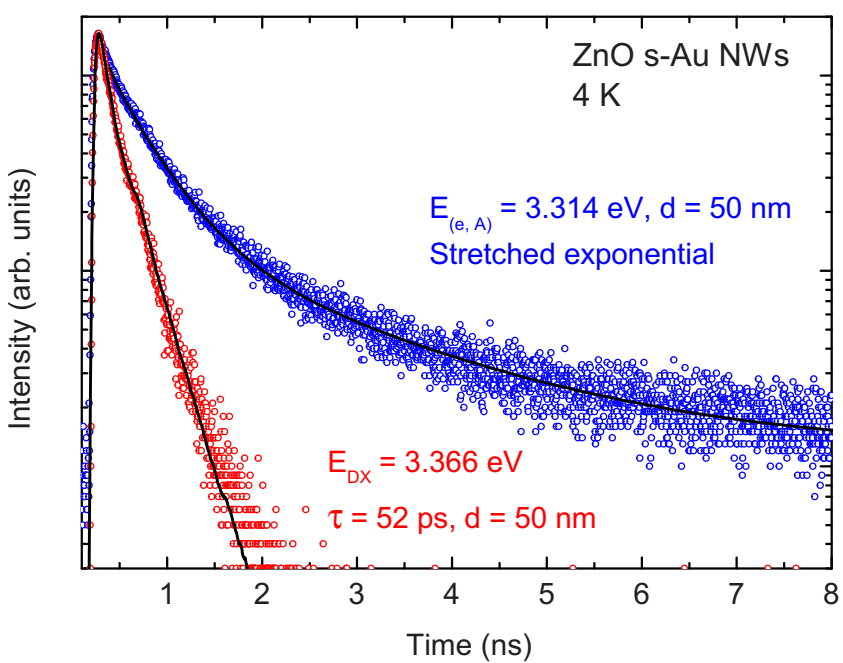

FIG. 3. (Color online) Representative low-temperature time resolved photoluminescence spectra at 3.366 and $3.314 \mathrm{eV}$ for s-Au NWs with $\mathrm{d}=50 \mathrm{~nm}$.

$3.366 \mathrm{eV}$ is somehow controversial as we have already previously discussed. ${ }^{4}$ Unfortunately, within the employed optical techniques we are unable to suggest its precise origin. Nevertheless, it is worth to mention that its linewidth and recombination time are similar to those observed for the $\mathrm{I}_{6}$ and $\mathrm{I}_{9}$ excitons (see Fig. 4). This suggests that this transition might be related to a DX impurity not identified up to date. In any case, independently of its precise origin its recombination dynamics will be similarly influenced by the NWs dimensions as we will shortly discuss.

Figure 3 shows representative transients from the DX at $3.366 \mathrm{eV}$ and of the free-to-bound transition at $3.314 \mathrm{eV}$ for the s-Au sample with $\mathrm{d}=50 \mathrm{~nm}$. The lifetime of the three DX lines is generally well described by a single exponential form, which is a signature of a two-level process as expected from these transitions. On the contrary, the decay time of the transition observed around $3.314 \mathrm{eV}$ is clearly not single exponential but rather well represented by a stretched exponential function. This result provides a confirmation that its origin is related to a distribution of states, in accordance with the free-to-bound assignment proposed by Schirra et al. ${ }^{6}$

We now turn to the discussion of the influence of finitesize on the recombination times of the DX lines around $3.360 \mathrm{eV}$. The dependence of the lifetimes with the length and diameter of the NWs is shown in Figs. 4(a) and 4(b), respectively. At this point we point out that the DX are spatially localized states since they arise from trapping of the free A-exciton to different localized impurity complexes. Consequently, these states exclusively probe the influence of the NW's finite-size on the lifetime of the optical modes which propagate through the NWs. In other words, the oscillator strength of the DX should not depend on the NWs dimensions directly but only through its influence on the confined electromagnetic field in the NWs. This would not be the case if investigating the dynamics of the free-exciton emission since these states have a well defined $\mathbf{k}$ in the reciprocal space, which is strongly dependent on the NW's shape. The most striking result in Fig. 4 concerns the measured absolute values for the lifetimes of the DX. For the smaller length and diameter, these lifetimes $(\approx 40 \mathrm{ps})$ are below the typical values measured in bulk samples (300-400 ps) by almost one order of magnitude. ${ }^{8}$ This result can cer- 

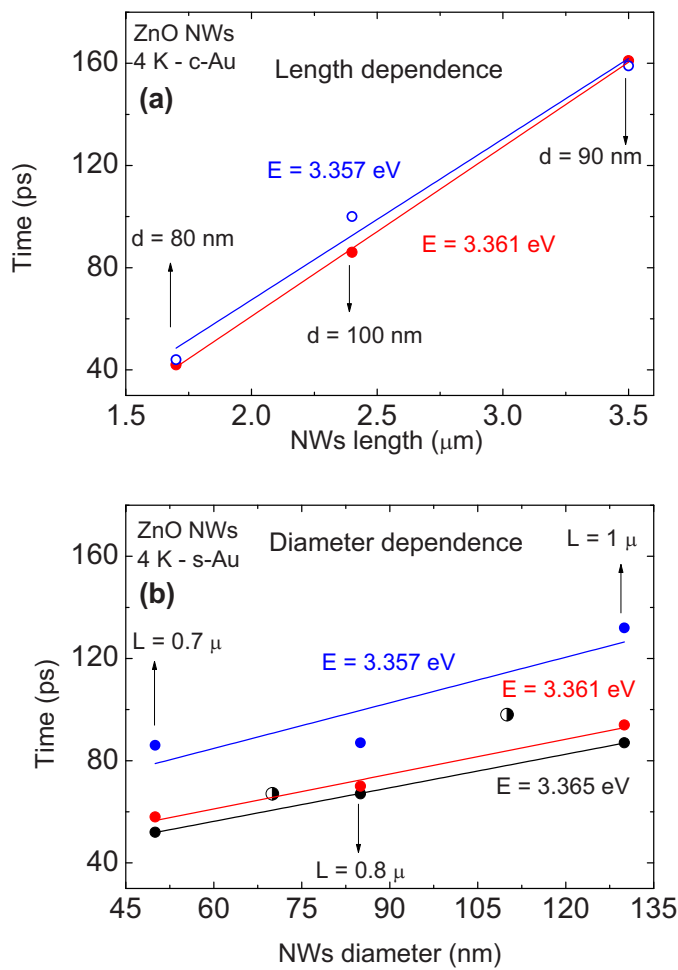

FIG. 4. (Color online) Lifetime of the different DX lines as a function of the NWs (a) length and (b) diameter. The half filled dots were extracted from Ref. 4.

tainly not be explained by a size-dependent $Q$-factor, whose effect is rather to increase the lifetime of the optical modes in the cavity. ${ }^{9}$ The origin of this effect is probably related to the presence of surface states, which act as alternative recombinations channels for the confined optical modes. Concerning the influence of the NWs length on the lifetimes, Fig. 4(a) shows larger lifetimes with increasing the length of the NWs, which can be understood by considering the dependence of the $Q$-factor on the NW's length. This dependence is by no means straightforward to obtain since the reflectivity at the ends of the NWs depends on size due to the diffraction caused by their subwavelength dimensions and, thus, cannot be calculated using the Fresnel relations. Following Ref. 10, the $Q$-factor is approximated by:

$$
Q=-L k_{z} / 2 \ln |r|,
$$

where $L$ is the NW's length, $k_{z}$ the propagation wave vector along the axis of the NWs, and $r$ is the reflectivity at the ends of the NWs (which is assumed to be equal for both ends). In addition, the lifetime of the optical modes confined in the NWs $\left(\tau_{\text {o.m }}\right)$ depends inversely on the confined mode linewidth $(\Delta f)$ as: $\tau_{o . m} \propto 1 / \Delta f \propto Q / f_{0}$ (with $Q \equiv f_{0} / \Delta f$ ). It follows that as $Q$ increases the lifetime of the optical modes in the cavity will also increase, which is reflected in the larger lifetimes (relative to the minimum value of about $40 \mathrm{ps}$ ) obtained for the DX in Fig. 4(a). Of course that the observed linear relation does not holds for smaller NW's lengths since it would lead to negative lifetimes. We point out that the influence of surfaces states on this series should be similar for all the samples since they have similar diameters (d $\approx 90 \mathrm{~nm}$ ).
The influence of the NW's diameter on the DX lifetimes is shown in Fig. 4(b). In this case, the length of the NWs is approximately constant $(L \approx 0.9 \mu)$, so no contribution of this term to the $Q$-factor is expected [see Eq. (1)]. Nevertheless, as reported in Ref. 11, a small contribution to the quality factor is expected with varying the NWs diameter. The main reason underlaying this effect relays on the fact that as the NW's diameter decreases, the optical mode spreads out of the NW's cross section. In fact, a smaller $Q$ is expected for the smaller diameters. Consequently, as varying the NW's diameter both effects (surfaces states recombinations and $Q$ enhancement) change continuously in the same direction, which prohibits decoupling these into separate contributions. For this purpose, the results in Fig. 4(a) provide valuable information since the surface states contribution can be considered as a constant for all the three samples. Finally, we can estimate the influence of each catalyst on the resulting surface states by comparing the similar samples in the two series (i.e., c-Au: $\mathrm{d}=80 \mathrm{~nm}, L=1.7 \mu$; and s-Au: d $=85 \mathrm{~nm}, L=0.8 \mu)$. The lifetimes for the corresponding sample in the c-Au series resulted in about $42 \mathrm{ps}$, whereas we found $70 \mathrm{ps}$ for the one of the c-Au series. Since both samples have approximately similar length, the contribution of the size dependent $Q$-factor is expected to be similar. Consequently, the observed smaller value for the c-Au sample suggests that the colloidal $\mathrm{Au}$ nanoparticles precursor introduces more surface states than the sputtered Au precursor. We are aware of the difference in length of these NWs, nevertheless, if a length correction was included the observed effect would be even larger.

In conclusion, we have shown that the DX constitute a unique tool to probe the finite-size influence on the lifetimes of the optical modes in $\mathrm{ZnO} \mathrm{NWs}$. We were able to partly decouple the influence of surface states from that of the mode quality factor, showing that the influence of these two effect is of the same order. Finally, we have shown that different catalysts introduce different surface states which might be of great importance for future applications based on these nanostructures.

This work was supported by the CONSOLIDER INGENIO 2009 CDS 00050 MULTICAT, by DFG within Grant No. SFB787 and by the "Unicat" cluster of excellence.

${ }^{1}$ A. B. Djurišić and Y. H. Leung, Small 2, 944 (2006).

${ }^{2}$ B. Gil and A. V. Kavokin, Appl. Phys. Lett. 81, 748 (2002).

${ }^{3}$ L. K. van Vugt, S. Rühle, P. Ravindran, H. C. Gerritsen, L. Kuipers, and D. Vanmaekelbergh, Phys. Rev. Lett. 97, 147401 (2006).

${ }^{4}$ J. S. Reparaz, F. Güell, M. R. Wagner, A. Hoffmann, A. Cornet, and J. R. Morante, Appl. Phys. Lett. 96, 053105 (2010).

${ }^{5}$ F. Güell, J. O. Ossó, A. R. Goñi, A. Cornet, and J. R. Morante, Nanotechnology 20, 315701 (2009).

${ }^{6}$ M. Schirra, R. Schneider, A. Reiser, G. M. Prinz, M. Feneberg, J. Biskupek, U. Kaiser, C. E. Krill, K. Thonke, and R. Sauer, Phys. Rev. B 77, 125215 (2008).

${ }^{7}$ B. K. Meyer, H. Alves, D. M. Hoffman, W. Kriegseis, D. Forster, F. Bertram, J. Christen, A. Hoffmann, M. Strassburg, M. Dworzak, U. Haboeck, and A. V. Rodina, Phys. Status Solidi B 241, 231 (2004).

${ }^{8}$ R. Heitz, C. Fricke, A. Hoffmann, and I. Broser, Mater. Sci. Forum 8387, 1241 (1992).

${ }^{9}$ V. Savona, L. C. Andreani, P. Schwendimann, and A. Quattropani, Solid State Commun. 93, 733 (1995).

${ }^{10}$ A. V. Maslov and C. Z. Ning, Appl. Phys. Lett. 83, 1237 (2003).

${ }^{11}$ M.-Q. Wang, Y.-Z. Huang, Q. Chen, and Z.-P. Cai, IEEE J. Quantum Electron. 42, 146 (2006). 